\title{
A Systematic Review of the Epidemiology, Immunopathogenesis, Diagnosis, and Treatment of Pleural TB in HIV-Infected Patients
}

\author{
A. Aljohaney, ${ }^{1,2}$ K. Amjadi, ${ }^{2}$ and G. G. Alvarez ${ }^{2}$ \\ ${ }^{1}$ Department of Internal Medicine, Faculty of Medicine, King Abdulaziz University, P.O. Box 80215, Jeddah 21589, Saudi Arabia \\ ${ }^{2}$ Divisions of Respirology and Infectious Diseases, Ottawa Hospital Research Institute, at the Ottawa Hospital, \\ University of Ottawa, 725 Parkdale Avenue, Ottawa, ON, Canada K1Y 4 E9
}

Correspondence should be addressed to G. G. Alvarez, galvarez@ohri.ca

Received 2 September 2011; Revised 14 December 2011; Accepted 29 December 2011

Academic Editor: Katalin Andrea Wilkinson

Copyright (C) 2012 A. Aljohaney et al. This is an open access article distributed under the Creative Commons Attribution License, which permits unrestricted use, distribution, and reproduction in any medium, provided the original work is properly cited.

\begin{abstract}
Background. High HIV burden countries have experienced a high burden of pleural TB in HIV-infected patients. Objective. To review the epidemiology, immunopathogenesis, diagnosis, and treatment of pleural TB in HIV-infected patients. Methods. A literature search from 1950 to June 2011 in MEDLINE was conducted. Results. Two-hundred and ninety-nine studies were identified, of which 30 met the inclusion criteria. The immunopathogenesis as denoted by cells and cytokine profiles is distinctly different between HIV and HIV-uninfected pleural TB disease. Adenosine deaminase and interferon gamma are good markers of pleural TB disease even in HIV-infected patients. HIV-uninfected TB suspects with pleural effusions commonly have a low yield of TB organisms however the evidence suggests that in dually infected patients smear and cultures have a higher yield. The Gene Xpert MTB/RIF assay has significant potential to improve the diagnosis of pleural TB in HIV-positive patients. Conclusions. Pleural TB in HIV-infected patients has a different immunopathogenesis than HIV-uninfected pleural TB and these findings in part support the differences noted in this systematic review. Research should focus on developing an interferon gamma-based point of care diagnostic test and expansion of the role of Gene Xpert in the diagnosis of pleural TB.
\end{abstract}

\section{Background}

The World Health Organization reported 1.1 million new cases of TB among HIV-infected persons in 2009 [1]. High HIV prevalence regions have experienced a greater burden of extrapulmonary TB [1]. Extrapulmonary TB is more common in HIV-infected patients compared with patients without HIV infection [2, 3] and its incidence has doubled since the beginning of the HIV pandemic [4]. Furthermore, pleural $\mathrm{TB}$ is the second most common form of extrapulmonary $\mathrm{TB}$ after peripheral lymph nodes in HIVinfected patients [4]. Pleural TB in the context of HIV continues to provide formidable challenges to clinicians around the world. In this systematic review, we report the epidemiology, immunopathogenesis, clinical presentation, diagnosis, and management of tuberculous pleural effusions in HIV-positive patients and explore some of the important differences between them and HIV-uninfected patients as well as how these findings could be applied in resource poor settings.

\section{Methods}

The PRISMA guidelines for systematic reviews [34] were used to formulate this paper.

2.1. Eligibility Criteria. Studies were included if all of the following criteria were met: (1) all study types except case reports published in English, French, Spanish, and Arabic and (2) pleural tuberculosis were defined by one of the following features: (a) positive culture of the pleural fluid or pleural biopsy specimen or both for M. tuberculosis, (b) positive sputum culture for M. tuberculosis and pleural effusion, positive acid fast bacilli (AFB) stain of the pleural fluid, or pleural biopsy specimen, or (c) granuloma on pleural biopsy with no alternative diagnosis and improvement on 
antituberculous therapy. (3) HIV infection documented by at least serological testing.

2.2. Search Strategy. A literature search in MEDLINE was conducted by one of the investigators (AA). Articles were limited to English, French, Spanish, and Arabic published between 1950 to June 2011. Using Boolean operator "and", we combined the following research themes (1) ("Tuberculosis, Pleural/complications" [Mesh] or "Tuberculosis Pleural/diagnosis" [Mesh] or "Tuberculosis, Pleural/ drug therapy" [Mesh] or "Tuberculosis, Pleural/enzymology" [Mesh] or "Tuberculosis, Pleural/epidemiology" [Mesh] or "Tuberculosis, Pleural/microbiology" [Mesh] or "Tuberculosis, Pleural/mortality" [Mesh] or "Tuberculosis, Pleural/pathology" [Mesh] or "Tuberculosis, Pleural/radiography" [Mesh] or "Tuberculosis, Pleural/surgery" [Mesh] or "Tuberculosis, Pleural/therapy" [Mesh]) or pleural tuberculosis [Text Word] and (2) "HIV Infections" [Mesh] or HIV infection [Text Word].

We also scanned the bibliographies of key articles to identify additional studies. The literature search was crosschecked by the university librarian to ensure reproducibility and that no other citations existed.

2.3. Study Selection and Data Collection Process. One reviewer (AA) screened the titles and abstracts of identified records. Articles were retrieved for full text review if they contained any information related to pleural tuberculosis and HIV infection. Full text review of these articles was performed by two reviewers (AA, GGA) independently using predefined case report forms. The two reviewers then met to discuss each article using the predefined case report forms. Differences were resolved by consensus. Variables that were collected included: incidence, prevalence, immunopathogenesis, diagnosis, management, and treatment of pleural TB in HIV-infected patients.

\section{Results}

3.1. Study Selection. Figure 1 outlines the identification, screening, eligibility, and inclusion of studies in this systematic review. The initial search strategy identified 295 potentially relevant articles and four additional studies from review of bibliographies. 63 articles were chosen based on titles and abstracts. 30 articles fulfilled the eligibility criteria and 33 articles were excluded after full text reviews. Selection and information bias, lack of uniform reporting, and inclusion of low methodological quality studies prevented a formal metaanalysis. Table 1 shows the characteristics of the 30 included studies.

\section{Epidemiology of Pleural TB in HIV-Infected Patients}

The prevalence rate of HIV-pleural tuberculosis was highest in the African regions compared to other regions (Table 2). The highest rate was in Zimbabwe where $85 \%$ of patients

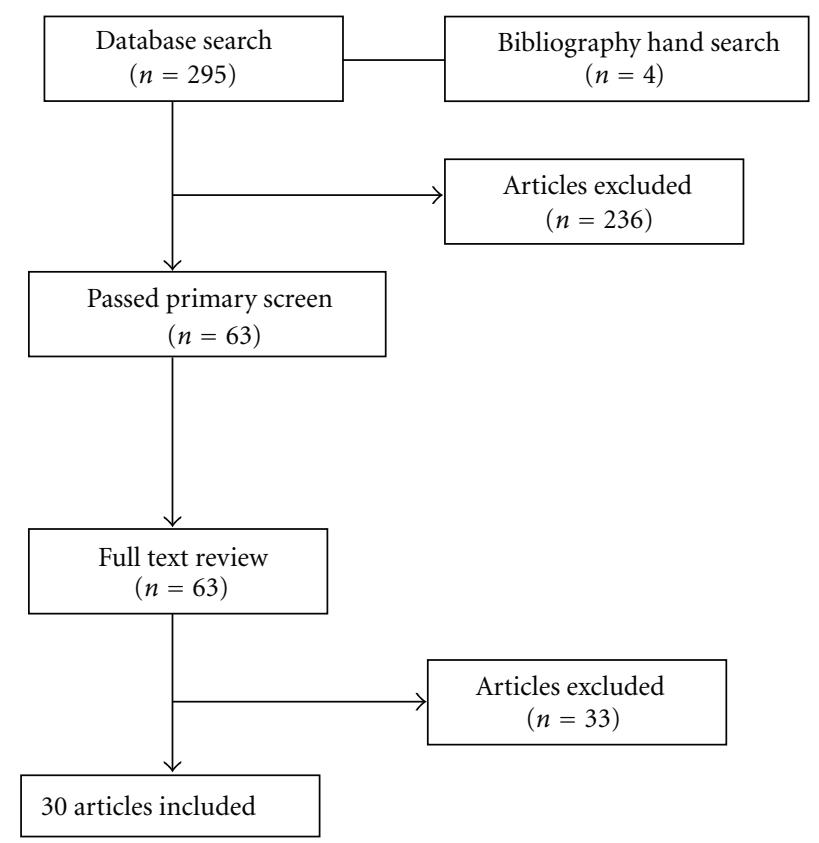

FIGURE 1: Identification, screening, eligibility, and inclusion of studies.

with tuberculous pleural effusions in one study were HIVpositive [16] and the lowest rate was seen in another study done in Spain at 10\% [15]. Although the study populations were heterogeneous, it can be extrapolated that due to the fact that the WHO estimates that $37 \%$ of dually infected incident cases came from the African region [1], it would be expected that more pleural TB would be seen in these regions. Males were more frequently infected with HIV and there was no gender differences noted between HIV-positive compared with HIV-uninfected individuals [18, 23, 24].

\section{Immunopathogenesis of Pleural TB in HIVInfected Patients}

Pleural TB has been characterized as a systemic inflammatory response which in some HIV-uninfected patients has been documented to resolve without the use of antibiotics [36]. This systemic inflammatory response likely contributes to the constellation of symptoms seen in these patients which can be more severe than in pulmonary TB [37]. The development of a pleural effusion in immunocompetent hosts is associated with an intense cell-mediated immune response with infiltration of CD4 $\mathrm{T}$ cells and production of high levels of proinflammatory cytokines such as gamma interferon (IFN-) and tumor necrosis factor alpha (TNF$\alpha)[36,38]$. In contrast, the immunopathogenesis of pleural $\mathrm{TB}$ in HIV-infected patients is different because of the CD4 $\mathrm{T}$ cell depletion and subsequent reduction in antigenspecific cytokine responses [39]. HIV-infected patients have decreased numbers s of CD4+ T cells and cytokine responses to TB [40-43]. When these immunological components are lacking the key protective immune response is significantly 
TABLE 1: Characteristics of included studies.

\begin{tabular}{|c|c|c|c|c|c|}
\hline \multirow{2}{*}{ Study/reference } & \multirow{2}{*}{ Year } & \multicolumn{2}{|c|}{ No of pleural TB patients } & \multirow{2}{*}{ Design } & \multirow{2}{*}{ Recruitment of patients } \\
\hline & & HIV+ & HIV- & & \\
\hline Aderaye et al. [5] & 1996 & 8 & 54 & Prospective cohort & Consecutive \\
\hline Batungwanayo et al. [6] & 1993 & 28 & 82 & Prospective cohort & Consecutive \\
\hline Cordero et al. [7] & 1995 & 12 & 107 & Retrospective chart review & N/A \\
\hline Dheda et al. [8] & 2009 & 38 & 13 & Prospective cohort & Consecutive \\
\hline Dheda et al. [9] & 2009 & 20 & 31 & Prospective cohort & Consecutive \\
\hline Domoua et al. [10] & 2007 & 30 & 17 & Prospective cohort & Consecutive \\
\hline Elliott et al. [11] & 1993 & 57 & 13 & Prospective cohort & Consecutive \\
\hline Frye et al. [2] & 1997 & 22 & 169 & Retrospective chart review & N/A \\
\hline Gil et al. [12] & 1995 & 10 & 93 & Retrospective chart review & $\mathrm{N} / \mathrm{A}$ \\
\hline Heyderman et al. [13] & 1998 & 63 & 11 & Prospective cohort & Consecutive \\
\hline Hodsdon et al. [14] & 2001 & 66 & 29 & Prospective cohort & Consecutive \\
\hline Luzze et al. [15] & 2001 & 109 & 33 & Prospective cohort & Consecutive \\
\hline Riantawan et al. [16] & 1999 & 37 & 52 & Prospective cohort & Consecutive \\
\hline Richter et al. [17] & 1994 & 65 & 47 & Prospective cohort & Consecutive \\
\hline Richter et al. [18] & 1994 & 49 & 26 & Prospective cohort & Consecutive \\
\hline Trajman et al. [19] & 1997 & 13 & 30 & $\begin{array}{l}\text { Cross-sectional } \\
\text { retrospective }\end{array}$ & N/A \\
\hline Villena et al. [20] & 1996 & 9 & 41 & Prospective cohort & N/A \\
\hline Baba et al. [21] & 2008 & 16 & 2 & Retrospective case series & N/A \\
\hline Baba et al. [22] & 2008 & 145 & 20 & Retrospective case control & $\mathrm{N} / \mathrm{A}$ \\
\hline Baba et al. [23] & 2008 & 5 & 23 & Prospective cohort & Consecutive \\
\hline Bezuidenhout et al. [24] & 2009 & 6 & 6 & Case series & N/A \\
\hline Collins et al. [25] & 2007 & 8 & 0 & Case series & N/A \\
\hline Conde et al. [26] & 2003 & 13 & 71 & Prospective cohort & Consecutive \\
\hline Hirsch et al. [27] & 2001 & 16 & 8 & Prospective cohort & Consecutive \\
\hline Jones et al. [28] & 2000 & 3 & 0 & Case series & $\mathrm{N} / \mathrm{A}$ \\
\hline Elliott et al. [29] & 2004 & 197 & 0 & $\begin{array}{l}\text { Randomized controlled } \\
\text { Trial }\end{array}$ & Consecutive \\
\hline Tshibwabwa-Tumba et al. [30] & 1997 & 159 & 68 & Prospective cohort & Consecutive \\
\hline Toossi et al. [31] & 2011 & 20 & 20 & Prospective cohort & Consecutive \\
\hline Siawaya et al. [32] & 2009 & 12 & 11 & Case series & N/A \\
\hline Mayanja-Kizza et al. [33] & 2009 & 20 & 0 & Case series & N/A \\
\hline
\end{tabular}

N/A: not mentioned in the methods section of the paper.

weakened. T helper 1 type cells secrete TNF $\alpha$, IFN $\gamma$, IL2, and IL12. These cells are important in the delayed type hypersensitivity reaction and in activating macrophages in pleural TB. Furthermore, granuloma formation is mediated by $\mathrm{CD} 4+\mathrm{T}$ cells of the $\mathrm{T}$ helper 1 type.

5.1. Histopathology. In an early study done in Tanzania [35], 36 HIV TB pleural biopsies were compared with 21 HIVuninfected TB-pleural biopsies. Histological characterization of the tissue reaction in pleural biopsies were examined as follows: reactive (well-formed granulomas with caseous necrosis, epitheliod cells, giant cells, scarce acid fast bacilli, or undetected $\mathrm{AFB}$ ), hyporeactive (poorly formed granulomas with noncaseous necrosis, few epithelioid cells or macrophages, and no giant cells, AFB were easily seen) and non-reactive (no true granuloma formation, noncaseous necrosis with nuclear debris and neutrophils, no giant cells and numerous AFB). Although CD4 counts were not done in this study, HIV-positive patients had significantly more pleural biopsies demonstrating the hyporeactive and nonreactive patterns than HIV-uninfected patients (14/36 versus $2 / 21 P<0.02$ ) and their level of immunodeficiency was worse than those with reactive patterns among the HIVinfected patients as evidenced by a greater number of AIDS 
TABLE 2: Reported prevalence rates of HIV-pleural tuberculosis across various studies/countries.

\begin{tabular}{lcc}
\hline Study/year & Country & Prevalence \\
\hline Heyderman et al. 1998 [13] & Zimbabwe & $85 \%$ \\
Batungwanayo et al. 1993 [6] & Rawanda & $80 \%$ \\
Luzze et al. 2001 [15] & Uganda & $80 \%$ \\
Domoua et al. 2007 [10] & Ivory Coast & $63 \%$ \\
Richter et al. 1994 [18] & Tanzania & $58 \%$ \\
Riantawan et al. 1999 [16] & Thailand & $37 \%$ \\
Elliott et al. 1993 [11] & Zambia & $31 \%$ \\
Trajman et al. 1997 [19] & Brazil & $30 \%$ \\
Aderaye et al. 1996 [5] & Ethiopia & $22 \%$ \\
Frye et al. 1997 [2] & USA & $11 \%$ \\
Gil et al. 1995 [12] & Spain & $10 \%$ \\
Cordero et al. 1995 [7] & Spain & $8 \%$ \\
\hline
\end{tabular}

related complications. Furthermore, hyporeactivity in the HIV group seemed to show a trend towards a mortality risk (3/11 versus $1 / 18$ deaths). In contrast, a case series of 12 patients (6 HIV-positive and $6 \mathrm{HIV}$ uninfected) more necrotic granulomas were seen in HIV-positive patients with pleural TB [24].

In a case series of three HIV-pleural TB cases [28], a significant number of mesothelial cells were noted in the pleural fluid. Commonly, few mesothelial cells are seen in the pleural space in patients with pleural TB since it is believed that there is extensive chronic inflammation that covers the mesothelium preventing it from exfoliating these cells into the pleural fluid.

5.2. Cytokine Profiles in Pleural TB in HIV-Infected Patients. Cytokine profiles in the pleural fluid of pleural TB in HIV-infected patients versus TB-pleural patients showed a mostly Th1 (or proinflammatory, instead of the Th2 or anti-inflammatory) cytokine profile [31]. No differences were found in the levels or patterns of cytokines in pleural fluid between HIV-positive and negative patients except for higher IL-8 levels seen in dually infected individuals [31]. In another study, comparing HIV-uninfected and HIVpositive pleural TB patients, 29 cytokines were measured in the plasma and pleural fluid. IL- $1 \beta$, IL-10, and TNF $\alpha$ were significantly decreased in the pleural fluid of HIV-positive pleural TB patients [32]. In addition, two proinflammatory markers, CXCL10/IP-10 and CCL3/MIP-1 $\alpha$, measured in the plasma were characteristic of pleural TB [32]. However, HIV infection affected the diagnostic accuracy as evidenced by a shift in cutoff values used, resulting in increased specificity at the expense of decreased sensitivity in pleural TB in HIV-infected persons compared to pleural TB in HIVnoninfected persons [32]. Other studies have shown that the levels of TNF $\alpha$ and MCP-1 were significantly elevated in the pleural fluid compared with autologous plasma in dually infected individuals [33]. MCP is a chemokine produced by fibroblasts and mesothelial cells and is a chemotactic agent for monocytes and lymphocytes. Both TB and HIV products can induce the production of MCP-1. Furthermore, transcription activation of HIV-1 in situ can be significantly reduced by neutralization of MCP-1; however, only when TNF $\alpha$. was not neutralized suggesting a possible relationship between MCP-1/TNF $\alpha$ [33].

Necrotic granulomas from pleural biopsies done in coinfected patients with pleural disease showed significantly elevated TNF $\alpha$-positive cells [24]. Although the authors acknowledged that this may not have equated to increased levels of the cytokine, the presence of this marker on the cells is an important difference which may explain the progression of pleural TB in HIV-infected patients in HIVinfected patients since apoptotic activity has been linked with TNF $\alpha$ and its continued presence is detrimental to the cellular environment [44] resulting in caseating necrosis. In another study, TNF $\alpha$ was elevated in HIV-pleural TB compared to HIV-uninfected pleural TB but the difference was not statistically significant [14]. In this same study, IFN $\gamma$ was significantly elevated in serum and pleural fluid in HIVpleural TB compared to HIV-uninfected pleural TB and it was suggested that CD8+ T cells could be the source which is supported by another study showing a relative increase in CD8+ T cells in HIV-positive patients with pleural TB [14]. However, mycobacterial replication was not controlled in the pleural space despite high levels of IFN $\gamma$. No difference was seen in the IL-10 levels in the pleural space between the two groups [14]. Another study [27] suggested apoptosis and levels of IFN $\gamma$ are increased in HIV-infected patients with pleural TB; however, this finding was not unique to HIV TB and seen in pleural TB alone.

\section{Clinical Features of Pleural TB in HIV-Infected Patients}

HIV-infected patients with pleural tuberculosis were more likely to present with fever, [13, 15, 17], dyspnea [17], cough [15] and significant weight loss [15] in comparison with HIV-uninfected patients. Furthermore, systemic symptoms and signs such as fatigue, night sweats, diarrhea, lymphadenopathy, splenomegaly, and hepatomegaly were more common in HIV-infected patients [13]compared to HIVuninfected patients [45]. Bilateral pleural effusions were also more frequently reported in HIV-positive patients [5] but the size or location of pleural effusions were comparable $[5,13,15]$. HIV-positive patients that present with pleural TB are generally sicker than non HIV-uninfected individuals as reflected by the increased frequency of systemic symptoms. Symptoms alone are limited in their ability to diagnose pleural TB in HIV-positive patients due to their non specific nature [13]. The severity of symptoms at presentation may reflect the higher degree of impairment in the immune system in HIV-positive patients which leads to more disseminated forms of the disease resulting in more advanced disease at presentation.

\section{Diagnosis}

7.1. Pleural Fluid. Pleural fluid examination showed increased mesothelial cells [28], decreased albumin and higher 
TABLE 3: Reported yields of different tests in HIV-infected patients with pleural TB.

\begin{tabular}{lcccccc}
\hline Study and year & $\begin{array}{c}\text { Pleural fluid } \\
\text { smear }\end{array}$ & $\begin{array}{c}\text { *Pleural fluid } \\
\text { culture }\end{array}$ & $\begin{array}{c}\text { Pleural biopsy } \\
\text { smear }\end{array}$ & $\begin{array}{c}* \text { Pleural biopsy } \\
\text { culture }\end{array}$ & $\begin{array}{c}\text { Pleural biopsy } \\
\text { histology }\end{array}$ & Sputum culture \\
\hline Elliott al. 1993 [11] & $0 \%$ & $26 \%$ & NR & $11 \%$ & $52 \%$ & NR \\
Richter et al. 1994 [18] & $6 \%$ & $43 \%$ & $20 \%$ & $35 \%$ & $86 \%$ & NR \\
Luzze et al. 2001 [15] & NR & $43 \%$ & NR & NR & $57 \%$ & NR \\
Conde et al. 2003 [26] & $8 \%$ & $15 \%$ & $38 \%$ & $77 \%$ & $92 \%$ & $77 \%$ \\
Hirsch et al. 2001 [27] & NR & $87 \%$ & NR & NR & NR & NR \\
Kitinya et al. 1993 [35] & $5 \%$ & $5 \%$ & $14 \%$ & $14 \%$ & NR & NR \\
Heyderman et al. 1998 & $18 \%$ & NR & $19 \%$ & $42 \%$ & $60 \%$ & NR \\
\hline [13]
\end{tabular}

* Reported yields of cultures using Löwenstein-Jensen medium.

NR: not referred for test.

gamma globulinlevels in HIV-infected patients [17]. Lymphocyte predominant effusions [15], LDH, protein and glucose levels did not differ between dually infected patients with pleural TB [16].

7.2. Mycobacterium TB Identification in Pleural Fluid. ZiehlNeelsen (ZN) stain $[13,15]$, liquid culture using BACTEC $[14,15]$ and Löwenstein-Jensen (LJ) cultures [11, 13, 15, $18,26,27,41]$ consistently provided a higher yield in HIVinfected individuals compared to HIV-uninfected individuals. In one study [13], the more immunocompromised the patient, the higher chance of finding $\mathrm{TB}$ organisms in the pleural fluid and the pleura itself. A CD4 count of $<200 \times 10^{6} / \mathrm{L}$ was associated with a positive pleural fluid smear $(37 \%$ versus $0 \% P=0.0006)$ and biopsy Ziehl-Neelsen stain (35\% versus 7\% $P=0.021)[13]$. The TB yield of different tests used in tuberculouspleuritis in HIV-infected patientsis shown (Table 3 ).

Although many studies looking at nucleic acid amplification tests (NAAT) applied to pleural TB in HIV-infected patients have been done [45] they have shown considerable variability. However, the new gene xpert MTB/RIF assay is a significant advance in point of care molecular diagnostic biology [46] that could provide a significant improvement in diagnosing pleural TB in HIV-infected patients with the added benefit that rifampin resistance is also detected by the assay and the result can be obtained in 2 hours. Although no studies have been published in HIV-infected patients with pleural $\mathrm{TB}$, a retrospective analysis of specimens sent to a national reference laboratory in Germany for mycobacteria [47] studied various specimen types including 113 pleural fluid samples for which the specificity was calculated at $98.1 \%$ and the sensitivity was not calculable.

7.3. HIV Identification in Pleural Fluid. HIV viral load in pleural fluid in dually infected patients has been demonstrated to be higher in the pleural fluid when compared to plasma [31]. Another study [33] showed that transcriptional activity of HIV-1 was significantly higher in pleural fluid mononuclear cells (PFMC) compared to peripheral blood mononuclear cells (PBMC). Increased HIV viral production was seen in the pleural space of HIV-pleural TB patients from activated HLA-DR mononuclear cells including lymphocytes and CD14+ macrophages [41]. Another study [25] suggested that HIV-positive patients with pleural TB showed higher HIV viral replication and heterogeneity which then migrated to the blood increasing systemic HIV heterogeneity. The excess viral loads seen in the pleural fluid of HIV-TBpleural patients make it an important site to gain a better understanding of whether the increased levels of virus seen in the pleural fluid of dually infected patients may affect HIV progression in these patients. The development of targeted inhibitors of viral replication in dually infected patients could offer new insight.

7.4. Pleural Biopsy. Ziehl-Neelsen stain of pleural biopsy carries significantly higher yield in HIV-infected patients than non HIV in two studies $[13,26]$ and not significantly higher in other studies $[17,35]$. The utility of histological examination of pleural biopsy was high in HIV-infected patients where granulomatous inflammation or caseous necrosis was detected in $52 \%-92 \%$. This finding was comparable with HIV-uninfected patients [11, 13, 15, 17, 26].

7.5. Sputum Culture. Sputum culture yield in pleural tuberculosis was reported to be higher in HIV-infected patients, however this was not statistically significant $[13,26]$. One of these studies [26], demonstrated that the yield of sputum cultures using sputum induction in dually infected patients with pleural TB who could not produce sputum spontaneously was high even in patients with no pulmonary findings on chest radiographs.

The use of the pleural fluid or biopsy smear to diagnose pleural TB in HIV-infected patientsremains an effective tool which is widely available and inexpensive especially in regions with limited resources. Clinicians often do not bother sending pleural fluid for smear and culture due to their low yield in the HIV-uninfected patient population; however, the evidence suggests that in dually infected patients smear and cultures should play an important role in the diagnostic process (Table 3). The pleural fluid and pleural biopsy TB culture yield is higher in HIV-infected patients. This may 
suggest that a higher bacillary burden is seen in the pleural space in HIV-infected patients because TB might cross from the lung parenchyma to the pleural space with greater ease as a result of an impaired immune response in the pleural space. Furthermore, the use of bedside inoculation of pleural fluid in BACTEC liquid medium provides a better sensitivity and faster results in HIV coinfection [15]. All of these classic forms of diagnosing pleural TB are limited in the prolonged time it takes to obtain results and also from the limited laboratory access in the clinic/hospital where the patients are assessed. These diagnostic delays result in significant morbidity and mortality of patients. In regions with limited resources, a point of care test which could be done at the bedside on pleural fluid would have a significant impact on the management and outcome of these patients.

7.6. Adenosine Deaminase ( $A D A)$. Adenosine deaminase (ADA) a $\mathrm{T}$ lymphocyte enzyme that catalyzes the conversion of adenosine and deoxyadenosine to inosine and deoxyinosine, respectively. Two different molecular forms of ADA, ADA 1, and ADA2 have been identified [48]. ADA1 is found in all cells, with its greatest activity in lymphocytes and monocytes. ADA2 isoenzyme is found mainly in monocytes/macrophages. Most of the ADA found in tuberculous pleural fluid is ADA2, whereas most of the ADA found in other pleural fluids is ADA1. Testing ADA levels in the pleural fluid is an easy, inexpensive, and useful test to establish the diagnosis of pleural TB. ADA retains its high utility in all HIV-infected patients [16] even patients with low CD4 counts [22]. ADA improves the accuracy of diagnosis in HIV-infected patients with pleural TB $[16,17$, 22]. In all HIV-infected patients regardless of CD4 counts, the sensitivity of ADA was 94\% when the cutoff value of $30 \mathrm{u} / \mathrm{l}$ was used and specificity of $95 \%$ [22]. The positive likelihood ratio was 18.9 and the negative likelihood ratio was 0.06 [22]. The sensitivity wasalso highat $96 \%$ when the cutoff value used was $60 \mathrm{u} / \mathrm{l}$ in HIV-infected patients [16]. These results were comparable to HIV-uninfected patients [16].

However, it should be noted that the sensitivity and specificity of ADA vary according to the different cutoff levels and also to different TB population prevalence. ADA measurement has a limited value in regions of low $\mathrm{TB}$ prevalence [49] as it can also be elevated in patients with empyema, lymphoma, lung cancer, rheumatoid arthritis, systemic lupus erythematosus, brucellosis, and Q fever [50]. In a high HIV and TB prevalence region, the use of ADA at a higher cut point ( $47 \mathrm{IU} / \mathrm{L})$ compared to the standard cut point $(30 \mathrm{IU} / \mathrm{L})$ in which a subgroup of HIV tested patients were studied, it was noted that ADA increases its specificity while use of the standard cut point ( 30 IU/L) results in loss of specificity but an increase in its sensitivity and thus improves its ability to rule out disease.

A possible explanation for the high levels of ADA even in HIV-infected patients with low CD4 counts may be related to the fact that monocytes are not significantly affected by HIV coinfection and they are the primary cells responsible for the production of isoenzyme ADA-2. Riantawan et al.
[16] documented the best cutoff of ADA at $60 \mathrm{U} / \mathrm{L}$ in HIVinfected patients which provides sensitivity of $95 \%$ and specificity of 96\%. Liang QL et al. [51] published a metaanalysis in mostly HIV-uninfected patients which included 63 studies documenting sensitivity and specificity of pleural $\mathrm{ADA}$ in the diagnosisof pleural $\mathrm{TB}$ to be 92 and $90 \%$, respectively [51]. The positive likelihood ratio was 9.03 , the negative likelihood ratio was 0.10 , and the diagnostic odds ratio was 110.08 [51]. The most widely accepted cutoff value for pleural fluid ADAis $40 \mathrm{U} / 1$ [50]; however, this cutoff will likely need to be higher in the HIV population.

7.7. Interferon Gamma. Interferon gamma levels in pleural tuberculosis were significantly higher in both serum and pleural fluid of HIV-positive patients when compared with HIV-uninfected patients [14]. There was no statistically significant correlation between blood CD4 cell count and the level of pleural INF-gamma $[14,21]$ but a positive correlation with pleural fluid viral loads was noted (correlation coefficient $0.54, P=0.02$ ) [14]. Another study documented interferon gamma sensitivity of $99 \%$ and specificity of $98 \%$ using $3.7 \mathrm{U} / \mathrm{mL}$ cutoff point which did not differ between HIV-positive and HIV-uninfected patients [20]. In a meta analysis of 27 studies evaluating the role of interferon gamma release assays (T-SPOT.TB1, QFT-G-IT), and Tuberculin Skin Test (TST) in diagnosing active tuberculosis [52], 5 studies used IGRA tests for the diagnosis of pleural TB $[8,21,53-55]$. None of these studies tested all of the patients entering the respective studies for HIV and all were limited by small sample sizes. However, one study [8] did test most of the cohort for HIV (51/67) and managed to confirm that even in a high HIV burden region, unstimulated pleural fluid interferon gamma levels measured in TB suspects were found to be highly sensitive and specific for distinguishing pleural TB from non-TB effusions [8]. In the same study it was also shown that because many HIV-positive patients likely have paucicellular pleural inflammation, adequate volumes of pleural fluid need to be obtained $(>20 \mathrm{~mL})$ in order to obtain adequate number of cells to analyze [8]. Interferon gamma inducible protein of $10 \mathrm{kDa}$ (IP-10) and lipoarabinomannan (LAM) mycobacterial antigen-detection assay were not useful in discriminating pleural TB patients versus non-TB patients in a subset of HIV-infected patients; however, future work should focus on validating IP-10 ability to rule out pleural TB [9]. The impact of immunosupression seen in HIV infection on the diagnostic accuracy of pleural mononuclear cells and their capacity to secrete interferon gamma requires further study in larger studies to confirm the accuracy of the findings presented.

Pleural TB in HIV-infected patients remains a challenge to diagnose because often sputum smears and cultures are negative. Thoracentesis is commonly performed to examine the composition of the pleural fluid. However, biochemical, histological and microbiological examination is usually limited in low-resource countries. A single point of care test that could be applied to pleural fluid would have enormous impact on the diagnosis and management of these cases. 


\section{Treatment}

There is no evidence to suggest that HIV-pleural tuberculosis should be treated differently than pulmonary TB. Two months of isoniazid, rifampin, pyrazinamide and ethambutol followed by 4 months of isoniazid and rifampin for susceptible organisms to all first line drugs are the international standard [56].

It is recommended that antiretroviral therapy should be delayed for 2 months unless the CD4 $<100 \times 10^{6} / \mathrm{L}$ to prevent immune reconstitution syndrome which could occur in one-third of patients [57]. The use of rifabutin instead of rifampin is recommended to avoid drug interaction with protease inhibitors and most nonnucleoside reverse transcriptase inhibitors, with the exception of efavirenz. Even so, rifampin-containing regimens can be prescribed if the selected antiretroviral drugs include efavirenz and two nucleoside transcriptase inhibitors (e.g., tenofovir and emtricitabine) [50]. In one randomized controlled study of 197 patients with HIV associated pleural TB, the administration of prednisolone as adjunctive therapy to standard first line TB therapy did not result in a survival benefit and although it was associated with faster resolution of the TB it was also associated with an increased risk of Kaposi sarcoma [29]. The patients in this study were significantly immunocompromised and the effects of steroids on HIVinfected patients with CD4 counts $>200 \times 10^{6} / \mathrm{L}$ still need to be assessed. A recent Cochrane review found no clear evidence to support the use of adjunctive corticosteroids in all persons with TB-pleural effusion [58].

\section{Quality of Included Studies}

Many of the studies had small sample sizes and did not have consecutive recruitment of patients which may have resulted in selection and information bias. Furthermore, the study design limited the quality of several studies with only one randomized controlled trial, several prospective cohorts, some retrospective chart reviews and several case series. Another significant limitation is the fact that many of the HIV-infected patients had different levels of immunosuppression (different levels of CD4 cells) which could affect the immunopathogenesis studies.

\section{Reasons for Exclusion of Studies}

Many of the studies that were excluded from this analysis did not establish the diagnosis of pleural TB or HIV accurately. One common reason for exclusion was that the patients in the HIV-uninfected group were not tested for HIV and assumed to be HIV uninfected. Some of the earlier studies opted to make a clinical diagnosis of HIV instead of getting serological testing which may have not been available at the time of the study. Other studies categorized pulmonary TB in the same category as pleural TB, whereas we categorized it as extrapulmonary disease with specific criteria depicted in the inclusion criteria section. Several studies were excluded because they were case reports.

\section{Future Research}

Pleural TB in HIV-infected patients has a different immunopathogenesis than HIV-uninfected pleural TB and these findings in part support the diagnostic differences seen in the yield of TB in the pleura in HIV-infected patients compared to HIV-uninfected patients. More research is needed in the field of immunopathogenesis of HIV-pleural TB disease as it offers an important microcosm where the bacteria and virus interact allowing the study of this complex interaction between HIV and TB to be further elucidated. Furthermore, the immunopathogenic differences may help develop a better interferon gamma-based point of care test that could be used at the bedside on pleural fluid in low-resource countries with high HIV prevalence. The Gene Xpert MTB/RIF assay technology applied to pleural fluid is a formidable area of research which could have enormous impact on the early diagnosis of pleural TB in HIV-positive patients and should be deemed a research priority in this field.

\section{Conflict of Interests}

No conflict of interest to declare by any of the authors.

\section{References}

[1] World Health Organization, WHO Report 2010 Global Tuberculosis Control, Journal [serial on the Internet], 2010, http://www.who.int/tb/publications/global_report/2010/ gtbr10_main.pdf.

[2] M. D. Frye, C. J. Pozsik, and S. A. Sahn, “Tuberculous pleurisy is more common in AIDS than in non-AIDS patients with tuberculosis," Chest, vol. 112, no. 2, pp. 393-397, 1997.

[3] J. M. FitzGerald, S. Grzybowski, and E. A. Allen, "The impact of human immunodeficiency virus infection on tuberculosis and its control," Chest, vol. 100, no. 1, pp. 191-200, 1991.

[4] S. K. Sharma and A. Mohan, "Extrapulmonary tuberculosis," Indian Journal of Medical Research, vol. 120, no. 4, pp. 316353, 2004.

[5] A. G. Aderaye, B. K. Melaku, and C. G. Zenebe, "Pleural tuberculosis in patients infected with HIV in Addis Ababa," Central African Journal of Medicine, vol. 42, no. 12, pp. 337340, 1996.

[6] J. Batungwanayo, H. Taelman, S. Allen, J. Bogaerts, A. Kagame, and P. van de Perre, "Pleural effusion, tuberculosis and HIV1 infection in Kigali, Rwanda," AIDS, vol. 7, no. 1, pp. 73-79, 1993.

[7] P. J. Cordero, V. Gil Suay, J. V. Greses et al., "The clinical characteristics of pleural tuberculosis in patients with and without human immunodeficiency virus infection," Archivos de Bronconeumología, vol. 31, no. 10, pp. 512-518, 1995.

[8] K. Dheda, R. N. van Zyl-Smit, L. A. Sechi et al., "Utility of quantitative T-cell responses versus unstimulated interferon- $\gamma$ for the diagnosis of pleural tuberculosis," European Respiratory Journal, vol. 34, no. 5, pp. 1118-1126, 2009.

[9] K. Dheda, R. N. Van-Zyl Smit, L. A. Sechi et al., "Clinical diagnostic utility of IP-10 and LAM antigen levels for the diagnosis of tuberculous pleural effusions in a high burden setting," PLoS ONE, vol. 4, no. 3, Article ID e4689, 2009.

[10] K. Domoua, T. Daix, G. Coulibaly et al., "Tuberculous pleural 
effusion and HIV infection at the pulmonary disease clinic in Abidjan, Ivory Coast," Revue de Pneumologie Clinique, vol. 63, no. 5, part 1, pp. 301-303, 2007.

[11] A. M. Elliott, B. Halwiindi, R. J. Hayes et al., "The impact of human immunodeficiency virus on presentation and diagnosis of tuberculosis in a cohort study in Zambia," Journal of Tropical Medicine and Hygiene, vol. 96, no. 1, pp. 1-11, 1993.

[12] V. Gil, P. J. Cordero, J. V. Greses, and J. J. Soler, "Pleural tuberculosis in HIV-infected patients," Chest, vol. 107, no. 6, pp. 1775-1776, 1995.

[13] R. S. Heyderman, R. Makunike, T. Muza et al., "Pleural tuberculosis in Harare, Zimbabwe: the relationship between human immunodeficiency virus, CD4 lymphocyte count, granuloma formation and disseminated disease," Tropical Medicine and International Health, vol. 3, no. 1, pp. 14-20, 1998.

[14] W. S. Hodsdon, H. Luzze, T. J. Hurst et al., "HIV-1-related pleural tuberculosis: elevated production of IFN- $\gamma$, but failure of immunity to Mycobacterium tuberculosis," AIDS, vol. 15, no. 4, pp. 467-475, 2001.

[15] H. Luzze, A. M. Elliott, M. L. Joloba et al., "Evaluation of suspected tuberculous pleurisy: clinical and diagnostic findings in HIV-1-positive and HIV-negative adults in Uganda," International Journal of Tuberculosis and Lung Disease, vol. 5, no. 8, pp. 746-753, 2001.

[16] P. Riantawan, P. Chaowalit, M. Wongsangiem, and P. Rojanaraweewong, "Diagnostic value of pleural fluid adenosine deaminase in tuberculous pleuritis with reference to HIV coinfection and a Bayesian analysis," Chest, vol. 116, no. 1, pp. 97-103, 1999.

[17] C. Richter, R. Perenboom, I. Mtoni et al., "Clinical features of HIV-seropositive and HIV-seronegative patients with tuberculous pleural effusion in dar es Salaam, Tanzania," Chest, vol. 106, no. 5, pp. 1471-1476, 1994.

[18] C. Richter, R. Perenboom, A. B. M. Swai et al., "Diagnosis of tuberculosis in patients with pleural effusion in an area of HIV infection and limited diagnostic facilities," Tropical and Geographical Medicine, vol. 46, no. 5, pp. 293-297, 1994.

[19] A. Trajman, E. B. Neto, M. T. C. T. Belo et al., "Pleural tuberculosis and human immunodeficiency virus co-infection," International Journal of Tuberculosis and Lung Disease, vol. 1, no. 6, pp. 498-501, 1997.

[20] V. Villena, A. López-Encuentra, J. Echave-Sustaeta, P. Martín-Escribano, B. Ortuño-de-Solo, and J. Estenoz-Alfaro, "Interferon- $\gamma$ in 388 immunocompromised and immunocompetent patients for diagnosing pleural tuberculosis," European Respiratory Journal, vol. 9, no. 12, pp. 2635-2639, 1996.

[21] K. Baba, S. Sørnes, A. A. Hoosen et al., "Evaluation of immune responses in HIV infected patients with pleural tuberculosis by the QuantiFERON TB-Gold interferon-gamma assay," BMC Infectious Diseases, vol. 8, article 35, 2008.

[22] K. Baba, A. A. Hoosen, N. Langeland, and A. M. Dyrhol-Riise, "Adenosine deaminase activity is a sensitive marker for the diagnosis of tuberculous pleuritis in patients with very low CD4 counts," PLoS ONE, vol. 3, no. 7, Article ID e2788, 2008.

[23] K. Baba, A. M. Dyrhol-Riise, L. Sviland et al., "Rapid and specific diagnosis of tuberculous pleuritis with immunohistochemistry by detecting Mycobacterium tuberculosis complex specific antigen MPT64 in patients from a HIV endemic area," Applied Immunohistochemistry and Molecular Morphology, vol. 16, no. 6, pp. 554-561, 2008.

[24] J. Bezuidenhout, T. Roberts, L. Muller, P. van Helden, and G. Walzl, "Pleural tuberculosis in patients with early HIV infection is associated with increased TNF-alpha expression and necrosis in granulomas," PLoS ONE, vol. 4, no. 1, Article ID e4228, 2009.

[25] K. R. Collins, M. E. Quiñones-Mateu, M. Wu et al., "Human immunodeficiency virus type 1 (HIV-1) quasispecies at the sites of Mycobacterium tuberculosis infection contribute to systemic HIV-1 heterogeneity," Journal of Virology, vol. 76, no. 4, pp. 1697-1706, 2002.

[26] M. B. Conde, A. C. Loivos, V. M. Rezende et al., "Yield of sputum induction in the diagnosis of pleural tuberculosis," American Journal of Respiratory and Critical Care Medicine, vol. 167, no. 5, pp. 723-725, 2003.

[27] C. S. Hirsch, Z. Toossi, J. L. Johnson et al., "Augmentation of apoptosis and interferon- $\gamma$ production at sites of active $M y$ cobacterium tuberculosis infection in human tuberculosis," Journal of Infectious Diseases, vol. 183, no. 5, pp. 779-788, 2001.

[28] D. Jones, T. Lieb, M. Narita, E. S. Hollender, A. E. Pitchenik, and D. Ashkin, "Mesothelial cells in tuberculous pleural effusions of HIV-infected patients," Chest, vol. 117, no. 1, pp. 289-291, 2000.

[29] A. M. Elliott, H. Luzze, M. A. Quigley et al., "A randomized, double-blind, placebo-controlled trial of the use of prednisolone as an adjunct to treatment in HIV-1-associated pleural tuberculosis," Journal of Infectious Diseases, vol. 190, no. 5, pp. 869-878, 2004.

[30] E. Tshibwabwa-Tumba, A. Mwinga, J. O. M. Pobee, and A. Zumla, "Radiological features of pulmonary tuberculosis in 963 HIV-infected adults at three Central African Hospitals," Clinical Radiology, vol. 52, no. 11, pp. 837-841, 1997.

[31] Z. Toossi, C. S. Hirsch, M. Wu, H. Mayanja-Kizza, J. Baseke, and B. Thiel, "Distinct cytokine and regulatory $\mathrm{T}$ cell profile at pleural sites of dual HIV/tuberculosis infection compared to that in the systemic circulation," Clinical and Experimental Immunology, vol. 163, no. 3, pp. 333-338, 2011.

[32] J. F. Djoba Siawaya, N. N. Chegou, M. M. V. D. Heuvel et al., "Differential cytokine/chemokines and KL-6 profiles in patients with different forms of tuberculosis," Cytokine, vol. 47, no. 2, pp. 132-136, 2009.

[33] H. Mayanja-Kizza, M. Wu, H. Aung et al., "The interaction of monocyte chemoattractant protein-1 and tumour necrosis factor- $\alpha$ in Mycobacterium tuberculosis-induced HIV-1 replication at sites of active tuberculosis," Scandinavian Journal of Immunology, vol. 69, no. 6, pp. 516-520, 2009.

[34] D. Moher, A. Liberati, J. Tetzlaff et al., "Preferred reporting items for systematic reviews and meta-analyses: the PRISMA statement," PLoS Medicine, vol. 6, no. 7, Article ID e1000097, 2009.

[35] J. N. Kitinya, C. Richter, R. Perenboom, H. Chande, and I. M. Mtoni, "Influence of HIV status on pathological changes in tuberculous pleuritis," Tubercle and Lung Disease, vol. 75, no. 3, pp. 195-198, 1994.

[36] P. F. Barnes, S. D. Mistry, C. L. Cooper, C. Pirmez, T. H. Rea, and R. L. Modlin, "Compartmentalization of a $\mathrm{CD}^{+}{ }^{+} \mathrm{T}$ lymphocyte subpopulation in tuberculous pleuritis," Journal of Immunology, vol. 142, no. 4, pp. 1114-1119, 1989.

[37] L. Qiu, L. D. Teeter, Z. Liu, X. Ma, J. M. Musser, and E. A. Graviss, "Diagnostic associations between pleural and pulmonary tuberculosis," Journal of Infection, vol. 53, no. 6, pp. 377-386, 2006.

[38] P. F. Barnes, S. J. Fong, P. J. Brennan, P. E. Twomey, A. Mazumder, and R. L. Modlin, "Local production of tumor necrosis factor and IFN- $\gamma$ in tuberculous pleuritis," Journal of Immunology, vol. 145, no. 1, pp. 149-154, 1990. 
[39] A. M. Elliott, T. J. Hurst, M. N. Balyeku et al., "The immune response to Mycobacterium tuberculosis in HIV-infected and uninfected adults in Uganda: application of a whole blood cytokine assay in an epidemiological study," International Journal of Tuberculosis and Lung Disease, vol. 3, no. 3, pp. 239247, 1999.

[40] F. G. Imperiali, A. Zaninoni, L. La Maestra, P. Tarsia, F. Blasi, and W. Barcellini, "Increased Mycobacterium tuberculosis growth in HIV-1-infected human macrophages: role of tumour necrosis factor- $\alpha$, Clinical and Experimental Immunology, vol. 123, no. 3, pp. 435-442, 2001.

[41] S. D. Lawn, S. T. Butera, and T. M. Folks, "Contribution of immune activation to the pathogenesis and transmission of human immunodeficiency virus type 1 infection," Clinical Microbiology Reviews, vol. 14, no. 4, pp. 753-777, 2001.

[42] N. Oyaizu, N. Chirmule, V. S. Kalyanaraman, W. W. Hall, R. A. Good, and S. Pahwa, "Human immunodeficiency virus type 1 envelope glycoprotein gp120 produces immune defects in $\mathrm{CD}^{+}$T lymphocytes by inhibiting interleukin 2 mRNA," Proceedings of the National Academy of Sciences of the United States of America, vol. 87, no. 6, pp. 2379-2383, 1990.

[43] S. Polyak, H. Chen, D. Hirsch, I. George, R. Hershberg, and K. Sperber, "Impaired class II expression and antigen uptake in monocytic cells after HIV-1 infection," Journal of Immunology, vol. 159, no. 5, pp. 2177-2188, 1997.

[44] K. Dheda, H. Booth, J. F. Huggett, M. A. Johnson, A. Zumla, and G. A. W. Rook, "Lung remodeling in pulmonary tuberculosis," Journal of Infectious Diseases, vol. 192, no. 7, pp. 1201-1210, 2005.

[45] M. Pai, L. L. Flores, N. Pai, A. Hubbard, L. W. Riley, and J. M. Colford Jr., "Diagnostic accuracy of nucleic acid amplification tests for tuberculous meningitis: a systematic review and metaanalysis," Lancet Infectious Diseases, vol. 3, no. 10, pp. 633-643, 2003.

[46] C. C. Boehme, P. Nabeta, D. Hillemann et al., "Rapid molecular detection of tuberculosis and rifampin resistance," The New England Journal of Medicine, vol. 363, no. 11, pp. 1005-1015, 2010.

[47] D. Hillemann, S. Rüsch-Gerdes, C. Boehme, and E. Richter, "Rapid molecular detection of extrapulmonary tuberculosis by the automated genexpert MTB/RIF system," Journal of Clinical Microbiology, vol. 49, no. 4, pp. 1202-1205, 2011.

[48] E. Pérez-Rodriquez and D. J. Castro, "The use of adenosine deaminase and adenosine deaminase isoenzymes in the diagnosis of tuberculous pleuritis," Current Opinion in Pulmonary Medicine, vol. 6, no. 4, pp. 259-266, 2000.

[49] A. R. J. van Keimpema, E. H. Slaats, and J. P. M. Wagenaar, "Adenosine deaminase activity, not diagnostic for tuberculous pleurisy," European Journal of Respiratory Diseases, vol. 71, no. 1, pp. 15-18, 1987.

[50] J. M. Porcel, “Tuberculous pleural effusion,” Lung, vol. 187, no. 5, pp. 263-270, 2009.

[51] Q. L. Liang, H. Z. Shi, K. Wang, S. M. Qin, and X. J. Qin, "Diagnostic accuracy of adenosine deaminase in tuberculous pleurisy: a meta-analysis," Respiratory Medicine, vol. 102, no. 5, pp. 744-754, 2008.

[52] M. Sester, G. Sotgiu, C. Lange et al., "Interferon- $\gamma$ release assays for the diagnosis of active tuberculosis: a systematic review and meta-analysis," European Respiratory Journal, vol. 37, no. 1, pp. 100-111, 2011.

[53] M. Losi, A. Bossink, L. Codecasa et al., "Use of a T-cell interferon- $\gamma$ release assay for the diagnosis of tuberculous pleurisy,"
European Respiratory Journal, vol. 30, no. 6, pp. 1173-1179, 2007.

[54] N. N. Chegou, G. Walzl, C. T. Bolliger, A. H. Diacon, and M. M. van den Heuvel, "Evaluation of adapted wholeblood interferon- $\gamma$ release assays for the diagnosis of pleural tuberculosis," Respiration, vol. 76, no. 2, pp. 131-138, 2008.

[55] L. N. Lee, C. H. Chou, J. Y. Wang et al., "Enzyme-linked immunospot assay for interferon-gamma in the diagnosis of tuberculous pleurisy," Clinical Microbiology and Infection, vol. 15, no. 2, pp. 173-179, 2009.

[56] R. Long, Canadian Tuberculosis Standards, Journal [serial on the Internet], 6th edition, 2007, http://www .phac-aspc.gc.ca/ tbpc-latb/pubs/pdf/tbstand07_e.pdf.

[57] S. D. Lawn, L. Myer, L. G. Bekker, and R. Wood, "Tuberculosisassociated immune reconstitution disease: incidence, risk factors and impact in an antiretroviral treatment service in South Africa," AIDS, vol. 21, no. 3, pp. 335-341, 2007.

[58] M. E. Engel, P. T. Matchaba, and J. Volmink, "Corticosteroids for tuberculous pleurisy," Cochrane Database of Systematic Reviews, no. 4, Article ID CD001876, 2007. 


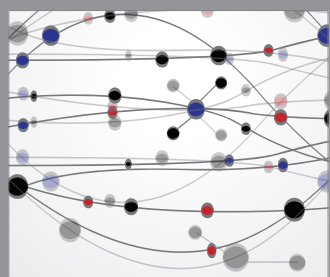

The Scientific World Journal
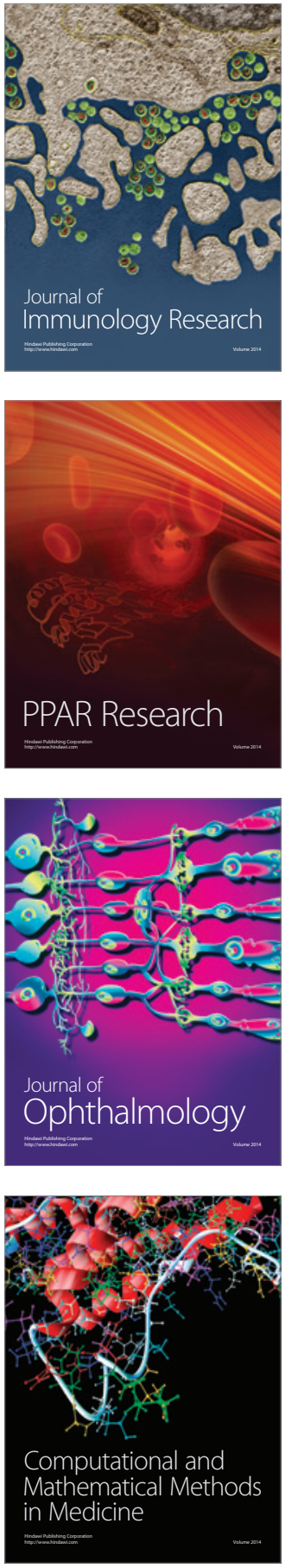

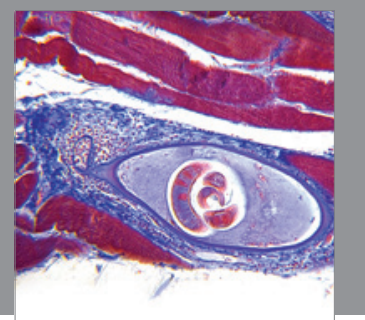

Gastroenterology

Research and Practice
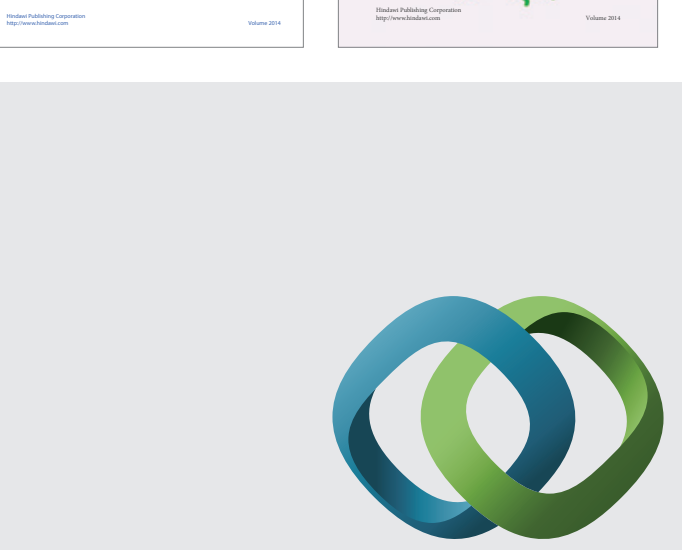

\section{Hindawi}

Submit your manuscripts at

http://www.hindawi.com
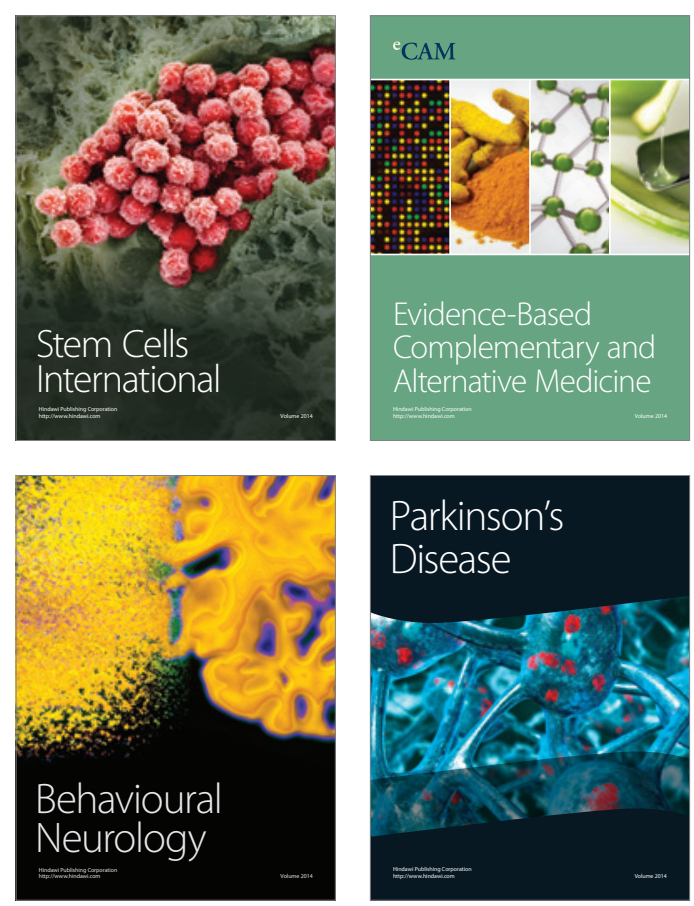

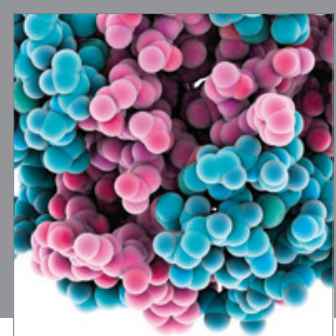

Journal of
Diabetes Research

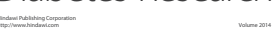

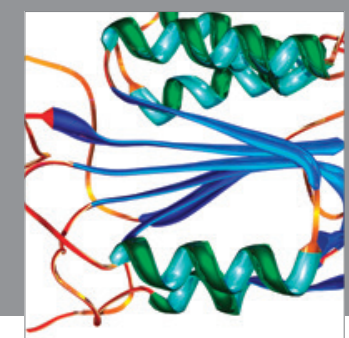

Disease Markers
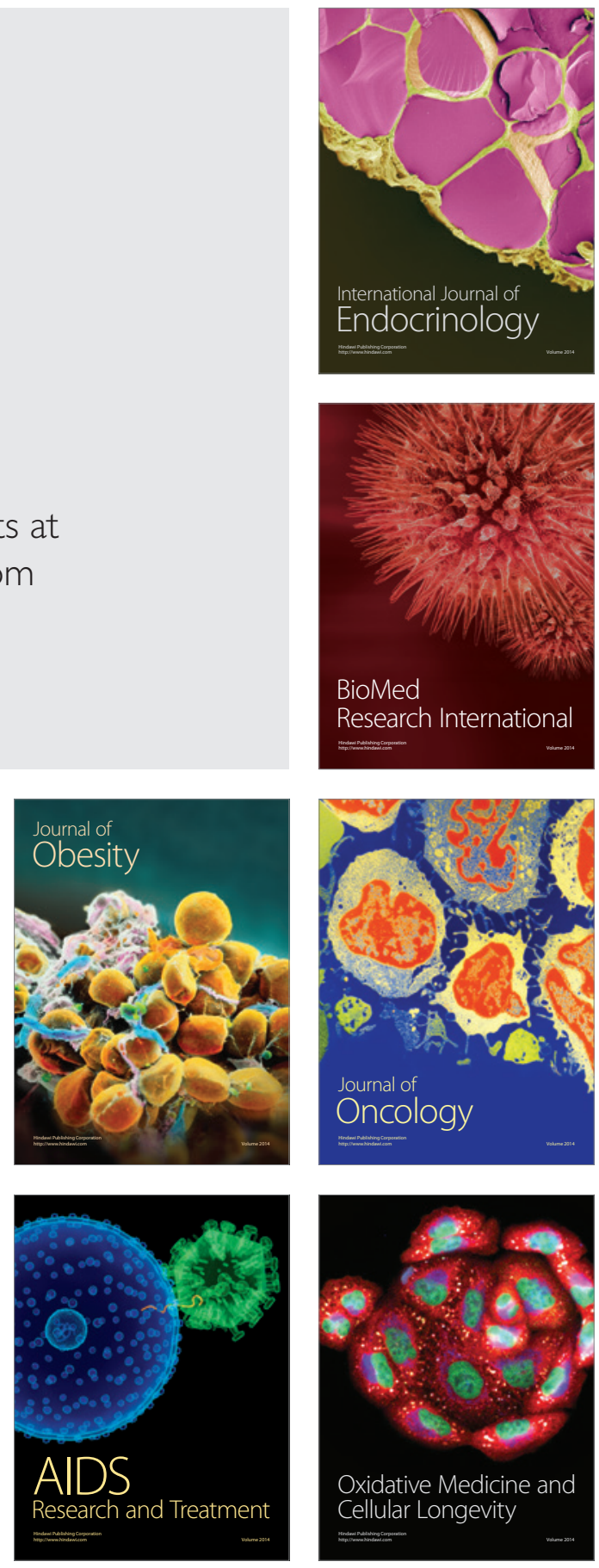